\title{
Carbon ion radiotherapy for locally advanced prostate cancer with bladder invasion: A case series report
}

\section{Yuhei Miyasaka}

Gunma University https://orcid.org/0000-0001-5137-1862

Hidemasa Kawamura ( $\nabla$ kawa@gunma-u.ac.jp )

https://orcid.org/0000-0003-4899-1715

Hiro Sato

Gunma University

Nobuteru Kubo

Gunma University

Tatsuji Mizukami

Toyama University

Hiroshi Matsui

Gunma University

Yoshiyuki Miyazawa

Gunma University

Kazuto Ito

Kurosawa hospital

Takashi Nakano

National Institutes for Quantum and Radiological Science and Technology

Kazuhiro Suzuki

Gunma University

Tatsuya Ohno

Gunma University

\section{Research}

Keywords: carbon ion radiotherapy, prostate cancer, bladder invasion

Posted Date: July 9th, 2020

DOI: https://doi.org/10.21203/rs.3.rs-40708/v1 
License: (c) (i) This work is licensed under a Creative Commons Attribution 4.0 International License. Read Full License 


\section{Abstract}

Background: The optimal management of clinical T4 (cT4) prostate cancer (PC) is still uncertain. At our institution, carbon ion radiotherapy (CIRT) with or without androgen-deprivation therapy (ADT) for nonmetastatic PC, including tumors invading the bladder, has been performed since 2010. Since carbon ion beams provide a sharp dose distribution with minimal penumbra and have biological advantages over photon radiotherapy, CIRT may provide a therapeutic benefit for PC with bladder invasion. Hence, we evaluated CIRT for locally advanced PC with bladder invasion in terms of the safety and efficacy.

Methods: Between March 2010 and December 2016, a total of 1,337 patients with nonmetastatic PC received CIRT at a total dose of $57.6 \mathrm{~Gy}$ (RBE) in 16 fractions over 4 weeks at our institution. Among them, 7 patients who had locally advanced PC with bladder invasion were identified. Long-term ADT was also administered to these patients. Adverse events were graded according to the Common Terminology Criteria for Adverse Event (CTCAE) version 5.0.

Results: At the conclusion of our study, all the patients with cT4 PC were alive with a median follow-up period of 78 months. Grade 2 acute urinary disorders were observed in only 1 patient. Regarding late toxicities, only 1 patient developed grade 2 hematuria and urinary urgency. There was no grade 3 or worse toxicity. Gastrointestinal toxicity was not observed. As for efficacy, $6(85.7 \%)$ patients had no recurrence or metastasis. One patient had biochemical and local failures 42 and 45 months after CIRT, respectively. However, the recurrent disease has been well controlled by salvage ADT.

Conclusions: Seven patients with locally advanced PC invading the bladder were treated with CIRT. There was no severe toxicity, and only 1 patient had clinical relapse, suggesting a positive safety profile for CIRT. Therefore, there is scope for future research among larger cohorts to ascertain the safety and effectiveness of CIRT for PC with bladder invasion.

\section{Background}

Localized prostate cancer $(\mathrm{PC})$ is generally treated with radical prostatectomy, external beam radiotherapy (EBRT), and brachytherapy, with or without androgen-deprivation therapy (ADT) [1]. Although favorable clinical outcomes following these treatments are well known, in the case of locally advanced PC invading adjacent structures, that is, clinical T4 (cT4) PC, the prognosis is not satisfactory [2]. A recent study reported that the addition of local therapy such as surgery and radiotherapy (RT) to systemic therapy, including ADT, provides a survival benefit even for cT4 PC [3]. Therefore, optimization of these local therapy is of importance in the management of cT4 PC.

Carbon ion radiotherapy (CIRT), which is one of the modality of EBRT initiated at the National Institute of Radiological Sciences in 1994 in Japan, provides a sharp dose distribution with minimal penumbra and has biological advantages owing to its high relative biological effectiveness (RBE) in the Bragg Peak, resulting from a high linear energy transfer [4]. At our institution, the Gunma University Heavy lon Medical Center (GHMC), CIRT for localized PC, including tumors invading bladder, has been performed since 
March 2010. Previous studies showed that CIRT for localized PC was a safe and effective treatment [58], but these studies did not include CT4 disease. Considering that CIRT has physical and biological advantages over photon radiotherapy, CIRT may provide therapeutic benefits even for the progressive PC. To evaluate this, we retrospectively reviewed patients with locally advanced PC with bladder invasion treated with CIRT.

\section{Methods}

\section{Patients}

Between March 2010 and December 2016, a total of 1,337 patients with clinically nonmetastatic PC received CIRT at our institution. All the patients were pathologically diagnosed with adenocarcinoma. $\square$ All pre-treatment biopsy specimens were re-evaluated by a central pathologist at Gunma University Hospital. Tumor grades were decided according to the modified Gleason grading system proposed by the International Society of Urological Pathology [9]. Urological examination, trans-rectal ultrasonography, computed tomography (CT), magnetic resonance imaging (MRI), and bone scintigraphy were performed for staging. Cystoscopic examination was also performed for patients with PC suspected of bladder invasion. Assessing these findings, the institutional cancer board with urological oncologists, radiologists, and radiation oncologists participated in diagnosing clinical stages of PC according to the International Union Against Cancer TNM classification (2002). In this study, we evaluated patients who had locally advanced PC with bladder invasion and without invasion to the rectum, pelvic floor muscles, and pelvic wall. Bladder invasion was diagnosed based on cystoscopic findings in principle; in cases when cystoscopy was not performed before ADT and when there were no apparent cystoscopic findings after $A D T, M R I$ findings before ADT were used for the diagnosis. All the treatment plans were approved by the institutional conference before carrying out the actual treatment.

\section{Carbon ion radiotherapy}

CIRT was performed at a total dose of $57.6 \mathrm{~Gy}$ (RBE) in 16 fractions over 4 weeks, with a fractional dose of $3.6 \mathrm{~Gy}$ (RBE) at 4 treatment sessions per week. Details of CIRT techniques have been previously reported [8]. The patients were positioned in a customized cradle (Moldcare; Alocare, Tokyo, Japan) with a low-temperature thermoplastic sheet (Shellfitter; Kuraray, Co., Ltd., Osaka, Japan). The bladder was filled with $100 \mathrm{~mL} 0.9 \%$ sterile saline and the rectum was emptied using enema just before CT simulation. Treatment planning was performed with Xio-N (Elekta, Stockholm, Sweden and Mitsubishi Electric, Tokyo, Japan) using a set of images of 2-mm-thick CT fused with MRI. Clinical target volume (CTV) included whole prostate, proximal seminal vesicle (SV), and bladder wall, which tumors invaded before ADT. For the tumor invading SV, CTV was expanded to include at least the invaded SV. The planning target volume (PTV1) for initial 9 fractions included CTV plus anterior and lateral margins of $10 \mathrm{~mm}$, cranial and caudal margins of $6 \mathrm{~mm}$, posterior margin of $5 \mathrm{~mm}$, and lateral margins to seminal vesicle of $3 \mathrm{~mm}$. The second PTV (PTV2) for the latter 7 fractions was generated by cutting the posterior PTV margin in front of the anterior wall of the rectum [6]. Each field was using a spread-out Bragg peak (SOBP), which was shaped 
with multi-leaf collimators and compensation bolus for each patient. Three radiation ports were used in the bilateral and anterior directions. At each treatment session using the anterior port, $\square$ the bladder was filled with $100 \mathrm{~mL} 0.9 \%$ sterile saline.

\section{Androgen deprivation therapy}

Long-term ADT was administered to all the patients with cT4 PC. Patients were treated with neoadjuvant combined androgen blockade therapy (CAB) consisting of luteinizing hormone-releasing hormone $(\mathrm{LH}-$ $\mathrm{RH}$ ) agonist or $\mathrm{LH}-\mathrm{RH}$ antagonist, and antiandrogen for at least 5 months before CIRT and during CIRT. After the completion of CIRT, all patients were treated with adjuvant LH-RH agonist or LH-RH antagonist monotherapy for a minimum total treatment period of 24 months.

\section{Followup and clinical evaluation}

All patients were followed up by physical examination and blood test, including PSA and urine test, at 3month intervals; CT, MRI, bone scintigraphy, and trans-rectal ultrasonography were performed once a year for 5 years. Adverse events (AE) were evaluated according to the Common Terminology Criteria for Adverse Events (CTCAE) version 5.0 [10]. Biochemical failure was defined in accordance with the Radiation Therapy Oncology Group-Association of Therapeutic Radiation Oncology Phoenix Consensus Conference definition [11].

\section{Results}

\section{Patients' characteristics}

Seven patients who had locally advanced PC with bladder invasion were identified from the medical record. The patients' characteristics are summarized in Table 1. The median follow-up period was 78 months (range, 37-109). The median age at diagnosis was 65 years (range, 53-81). The median initial prostate specific antigen (PSA) level was $32.1 \mathrm{ng} / \mathrm{ml}$ (range, $7.8-87.0)$. Three patients (42.9\%) had primary Gleason pattern 5 . Five patients $(71 \%)$ had seminal vesicle invasion. Three patients were diagnosed with bladder invasion by cystoscopic findings, while 4 patients were diagnosed by MRI findings. The median total duration of the ADT was 32 months (range, 24-46). 
Table 1

Summary of the patients' characteristics.

\begin{tabular}{|llllllll|}
\hline Patient number & $\mathbf{1}$ & $\mathbf{2}$ & $\mathbf{3}$ & $\mathbf{4}$ & $\mathbf{5}$ & $\mathbf{6}$ & $\mathbf{7}$ \\
\hline Age (years) & 53 & 72 & 61 & 74 & 81 & 65 & 57 \\
\hline Seminal vesicle invasion & + & + & - & + & - & + & + \\
\hline Gleason score & $5+4$ & $4+3$ & $4+3$ & $5+4$ & $4+5$ & $5+4$ & $4+5$ \\
\hline Positive cores & $8 / 8$ & $10 / 10$ & $4 / 8$ & $6 / 10$ & $8 / 8$ & $10 / 12$ & $10 / 12$ \\
\hline Initial PSA (ng/mL) & 11.6 & 87 & 37.3 & 32.1 & 73.7 & 7.8 & 9.39 \\
\hline ADT duration before CIRT (months) & 12 & 5 & 6 & 6 & 6 & 6 & 12 \\
\hline Total ADT duration (months) & 40 & 24 & 28 & 32 & 25 & 46 & 33 \\
\hline $\begin{array}{l}\text { Abbreviations: PSA = Prostate specific antigen, ADT = Androgen deprivation therapy, CIRT = Carbon ion } \\
\text { radiotherapy }\end{array}$ & & & & & \\
\hline
\end{tabular}

\section{Clinical Outcomes}

The clinical courses of the patients with locally advanced PC are summarized in Table 2. All the patients are alive and being followed-up. There was no grade 3 or worse AE. Acute urinary disorder was seen in 4 patients (\#2, \#3, \#6,\#7). One of these patients (\#3) needed alpha-blocker for urinary frequency. As for late toxicity, one patient (\#6) complained about urinary urgency, thus requiring medication. The patient took aspirin and developed hematuria 16 months after receiving CIRT. However, this AE was easily dealt by using a hemostatic agent (carbazochrome sodium sulfonate hydrate) and was never observed again. Two other patients have also taken medication, which increased the risk of bleeding (cilostazol and ethyl icosapentate), but they had no hematuria induced by CIRT. There were no gastrointestinal AE in these seven patients.

There was only one recurrence. A patient (\#2) had biochemical and local failure 42 and 45 months after $\mathrm{CIRT}$, respectively. The recurrent tumor was detected at the original site. There was no metastatic disease. Salvage CAB was administered to the patient, after which the recurrent disease was undetected on MRI, and serum PSA level monotonically decreased to under $0.1 \mathrm{ng} / \mathrm{ml}$ and remained low thereafter. The other patients have had no evidence of the disease. 
Table 2

Summary of the clinical course.

\begin{tabular}{|llllllll|}
\hline Patient number & 1 & 2 & 3 & 4 & 5 & 6 & 7 \\
\hline Follow-up (months) & 109 & 96 & 96 & 78 & 66 & 66 & 37 \\
\hline Alive/ Dead & Alive & Alive & Alive & Alive & Alive & Alive & Alive \\
\hline Biochemical failure & - & + & - & - & - & - & - \\
\hline Local failure & - & + & - & - & - & - & - \\
\hline Distant failure & - & - & - & - & - & - & - \\
\hline Acute toxicity (max grade) & & & & & & & \\
\hline Genitourinary & 0 & 1 & 2 & 0 & 0 & 1 & 1 \\
\hline Gastrointestinal & 0 & 0 & 0 & 0 & 0 & 0 & 0 \\
\hline Late toxicity (max grade) & & & & & & & \\
\hline Genitourinary & 0 & 0 & 1 & 0 & 0 & 2 & 1 \\
\hline Gastrointestinal & 0 & 0 & 0 & 0 & 0 & 0 & 0 \\
\hline
\end{tabular}

A representative case: Patient \#1

On July of 20XX, a 53-year-old Japanese man diagnosed with PC was referred to our institution since he desired to receive CIRT. At that time, approximately 10 months of $C A B$ consisting of leuprorelin acetate and bicalutamide had already reduced serum PSA from $11.8 \mathrm{ng} / \mathrm{mL}$ (September of 20XX-1) to < $0.01 \mathrm{ng} / \mathrm{mL}$ (August of 20XX), but cystoscopic findings clearly showed tumor invading the bladder neck. The institutional cancer board diagnosed the clinical stage as cT4NOMO by checking the CT images, MRI, bone scintigraphy, and cystoscopic findings (Fig. 1A, D). The tumor also invaded the right seminar vesicle. Pre-treatment biopsy specimens were reviewed by a central pathologist. Tumor cells were found in all the cores $(8 / 8)$ and the Gleason score was diagnosed as $5+4=9$.

CIRT was performed at a total dose of $57.6 \mathrm{~Gy}$ (RBE) in 16 fractions over 4 weeks from October to November of 20XX. Figure 2 shows the dose distribution. DDuring this treatment period, dermatitis (grade 1) was observed in the irradiated region. There was no other acute toxicity.

After completion of CIRT, bicalutamide was discontinued. Blood and urine tests were performed every 3 months and CT, MRI, bone scintigraphy, and trans-rectal ultrasonography were performed once a year for 5 years. Chronological changes in MRI findings are shown in Fig. 1B, C. Serum PSA levels were kept under $0.01 \mathrm{ng} / \mathrm{mL}$ till ADT was finished. The cystoscopic findings on April of 20XX +2 showed that the bladder lesion shrank but remained (Fig. 1E). Six months later, as similar findings were found in the cystoscopic examination, a transurethral resection biopsy was performed. The biopsy findings showed urothelial mucosa with xanthogranulomatous lesions and no malignant cells (Fig. 1F). After discussion 
with urological oncologists in Gunma University Hospital, leuprorelin acetate was discontinued on December of 20XX +2 . Thereafter, the serum PSA level was still well controlled. Approximately 9 years after CIRT, there have been no findings suggesting recurrence or metastasis. No late toxicity was observed.

\section{Discussion}

In the management of cT4 PC, the addition of local therapy to systemic therapy was associated with improved survival when compared to systemic therapy alone [3], but the optimal local therapy has not yet been established. We have treated locally advanced PC invading the bladder with CIRT with long-term ADT, expecting that the physical and biological advantages of CIRT over photon RT would yield therapeutic benefits. Thus, we evaluated the safety and efficacy of the CIRT in the current study. To the best of our knowledge, this is the first report describing CIRT with long-term ADT for locally advanced PC with bladder invasion. Our findings showed that none of the 7 patients had severe toxicity, and $6(85.7 \%)$ patients had no recurrence or metastasis with the median follow-up period of 78 months.

There are limited literatures on the surgery for cT4 PC. Hajili et al. showed that the prostate cancerspecific survival (PCSS) rates for CT4 PC at 150 months after inductive ADT and subsequent RP were $82 \%$, and $10.3 \%$ of the patients had complications requiring surgical intervention [12]. Kumazawa et al. reported $\square$ cystoprostatectomy $\square$ followed by immediate hormone therapy for cT4NOMO disease. In their study, the PCSS rate at 5 years after the surgery was $87.1 \%$ [13]. These findings showed relatively favorable survival despite the advanced disease, although it should be noted that these surgical indications were limited to patients with good general conditions.

EBRT, which is a less invasive treatment modality compared to surgery, is also recommended for very high risk PC including cT4 disease [1]. Furthermore, intensity-modulated radiotherapy (IMRT) and imageguided radiotherapy enable higher dose to tumors with lower dose to organs at risk, resulting in the lower incidence of AE and improved biochemical relapse free survival (bRFS) [14]. In addition, EBRT with highdose-rate brachytherapy boost may improve bRFS [15]. To our knowledge, little is known regarding the outcomes of patients with CT4 PC treated with EBRT, although a clinical trial to analyze whether surgical treatment or EBRT using photons is the better treatment for CT4 PC is undergoing [16].

CIRT, a kind of EBRT, contributes to favorable outcomes especially in advanced PC. Kasuya et al. reported that the prostate cancer specific mortality at 5 years after CIRT with long-term ADT was $1.5 \%$ for high risk PC [17]. We previously reported that the 5-year biochemical relapse-free rate of high risk PC was $92.0 \%$ in a single-institutional prospective study [8]. The present study showed that $85.7 \%(6 / 7)$ of the patients had no biochemical failure and all the patients were alive at the median follow-up period of 78 months. We cannot compare these results with those of EBRT due to the lack of available literature specific to cT4 PC, but when compared with the surgical treatment options, our results seem to be favorable, although we acknowledge that the number of patients included in our study is extremely small. 
In general, CIRT is also remarkable for the low incidence of late toxicity because of the superior dose accumulation. We previously demonstrated that $9 \%$ of the patients had grade 2 late toxicities after CIRT [8], while Cahlon et al. showed that up to $23 \%$ of the patients had grade 2 late toxicities after photonbased IMRT [18]. In CIRT for PC with bladder invasion, the irradiated volume of the bladder was larger than that in PC without bladder invasion, which potentially increases the incidence and severity of urinary toxicity. However, with the careful management of inter-fractional displacements mentioned above, there was only 1 patient with grade 2 late urinary disorder in the current study, thus supporting that CIRT is tolerable for patients with locally advanced PC with bladder invasion.

These favorable outcomes of the present study may be due to the physical and biological advantages of CIRT over photon RT, which may have provided therapeutic benefits for locally advanced PC. Although our findings provide only the weakest evidence, we are encouraged to further explore the safety and efficacy of CIRT for PC with bladder invasion in larger cohorts.

The present study has some limitations. As mentioned above, this is a case series report with extremely small number of patients; thus, some potential sources of bias were not excluded. In addition, the effects of clinical and pathological factors such as age, initial PSA level, Gleason score, the number of positive cores in biopsy samples, and the duration of ADT were not argued in this study. Larger cohort is needed to evaluate these factors.

\section{Conclusions}

In summary, we report 7 patients with locally advanced PC with bladder invasion who received CIRT with longterm $A D T$, with well tolerable toxicity and favorable prognoses. Our study provides only the weakest evidence because of the extremely small study population, but CIRT with long-term ADT seems to be a potential treatment option. For more reliable evidence, further studies are required.

\section{Abbreviations}

AE: adverse event; ADT: androgen-deprivation therapy; bRFS: biochemical relapse free survival; $C T$ : computed tomography; CAB: combined androgen blockade; CIRT: carbon ion radiotherapy; CTCAE: the Common Terminology Criteria for Adverse Event; CTV: clinical target volume; cT4: clinical T4; EBRT: external beam radiotherapy; GHMC: Gunma University Heavy Ion Medical Center; IMRT: intensitymodulated radiotherapy; PC: prostate cancer; LH-RH: luteinizing hormone-releasing hormone; MRI: magnetic resonance imaging; RBE: relative biological effectiveness; RT: radiotherapy; PCSS: prostate cancer-specific survival; PSA: prostate specif ic antigen; PTV: planning target volume; SOBP: spread-out Bragg peak; SV: seminal vesicle

\section{Declarations}

\section{Ethics approval and consent to participate}


The current study was designed and conducted according to the principles outlined in the Declaration of Helsinki and within the guidelines of Good Clinical Practices and was approved by the Institutional Review Board of Gunma University. The need for written informed consent was waived but all the patients in the study had the opportunity to opt out.

\section{Consent for publication}

Not applicable.

\section{Availability of data and material}

All the dataset is included within the article.

\section{Competing interests}

KS has received research grants from Takeda and Astellas.

\section{Funding}

No funding was obtained for this study.

\section{Authors' contributions}

HK, TN, KS, and TO designed, directed, and coordinated this study. YM (Yuhei Miyasaka), HS, and TM performed data collecting. YM (Yuhei Miyasaka) wrote the draft. HK, HS, NK, YM (Yoshiyuki Miyazawa), $\mathrm{HM}, \mathrm{KI}$, and KS suggested corrections and/or improvements. All authors have read and approved the manuscript.

\section{Acknowledgements}

None.

\section{References}

1. National Comprehensive Cancer Network (NCCN). Guidelines; Prostate Cancer version 2.2019 [Internet]. 2019 [cited 2019 Jul 18]. Available from: https://www.nccn.org/professionals/physician_gls/pdf/prostate.pdf.

2. $10.1016 /$ j.juro.2010.04.010 Hsiao W, Moses KA, Goodman M, Jani AB, Rossi PJ, Master VA. Stage IV prostate cancer: Survival differences in clinical t4, nodal and metastatic disease. J Urol [Internet]. Elsevier Inc.; 2010;184:5128. Available from: http://dx.doi.org/10.1016/j.juro.2010.04.010.

3. 10.1016/j.urology.2018.06.038

Kim AH, Konety B, Chen Z, Schumacher F, Kutikov A, Smaldone M, et al. Comparative effectiveness of local and systemic therapy for t4 prostate cancer. Urology [Internet]. Elsevier Inc.; 2018;120:173-9. 
Available from: https://doi.org/10.1016/j.urology.2018.06.038.

4. Kanai T, Endo M, Minohara S, Miyahara N, Koyama-Ito H, Tomura H, et al. Biophysical characteristics of HIMAC clinical irradiation system for heavy-ion radiation therapy. Int J Radiat Oncol Biol Phys [Internet]. 1999 [cited 2018 Apr 17];44:201-10. Available from: https://ac.elscdn.com/S0360301698005446/1-s2.0-S0360301698005446-main.pdf?_tid=6006fc12-d450-478f93fa-be28169b624d\&acdnat=1523962108_ac3601d6630bc4b5d36c925deff949ca.

5. Tsuji H, Yanagi T, Ishikawa H, Kamada T, Mizoe JE, Kanai T, et al. Hypofractionated radiotherapy with carbon ion beams for prostate cancer. Int J Radiat Oncol Biol Phys [Internet]. 2005 [cited 2019 Feb 26];63:1153-60. Available from: https://ac.els-cdn.com/S0360301605007091/1-s2.0S0360301605007091-main.pdf?_tid=0e60706d-3bbe-4a17-a6749ec39ab4457d\&acdnat=1551107039_a0b1f9a6766163176dbe5ef1e0e641e0.

6. Okada T, Tsuji H, Kamada T, Akakura K, Suzuki H, Shimazaki J, et al. Carbon ion radiotherapy in advanced hypofractionated regimens for prostate cancer: from 20 to 16 fractions radiation oncology. Int J Radiat Oncol Biol Phys [Internet]. 2012;84:968-72. [cited 2019 Feb $26 ;.$. Available from: www.redjournal.org.

7. Ishikawa H, Tsuji H, Kamada T, Yanagi T, Mizoe JE, Kanai T, et al. Carbon ion radiation therapy for prostate cancer: Results of a prospective phase II study. Radiother Oncol [Internet]. 2006;81:57-64. [cited 2019 Feb 26];: Available from: www.thegreenjournal.com.

8. Kawamura H, Kubo N, Sato H, Mizukami T, Katoh H, Ishikawa $\mathrm{H}$, et al. Moderately hypofractionated carbon ion radiotherapy for prostate cancer; a prospective observational study "GUNMA0702." BMC Cancer. BMC Cancer. 2020;20:75.

9. Epstein JI, Allsbrook WC, Amin MB, Egevad LL, Bastacky S, López Beltrán A, et al. The 2005 International Society of Urological Pathology (ISUP) consensus conference on Gleason grading of prostatic carcinoma. Am J Surg Pathol. 2005;29:1228-42.

10. NCl Common Terminology Criteria for Adverse Events. (CTCAE) v5.0 data files [Internet]. [cited 2020 May 31]. Available from: https://evs.nci.nih.gov/ftp1/CTCAE/About.html.

11. Roach M, Hanks G, Thames H, Schellhammer P, Shipley WU, Sokol GH, et al. Defining biochemical failure following radiotherapy with or without hormonal therapy in men with clinically localized prostate cancer: Recommendations of the RTOG-ASTRO Phoenix Consensus Conference. Int J Radiat Oncol [Internet]. 2006 [cited 2019 Jul 1];65:965-74. Available from: https://linkinghub.elsevier.com/retrieve/pii/S0360301606006638.

12. Hajili T, Ohlmann CH, Linxweiler J, Niklas C, Janssen M, Siemer S, et al. Radical prostatectomy in T4 prostate cancer after inductive androgen deprivation: results of a single-institution series with longterm follow-up. BJU Int [Internet]. 2019;123:58-64. [cited 2019 Jun 21];. Available from: www.bjui.org.

13. Kumazawa T, Tsuchiya N, Saito M, Inoue T, Narita S, Horikawa Y, et al. Cystoprostatectomy as a treatment of prostate cancer involving the bladder neck. Urol Int [Internet]. 2009;83:141-5. Available from: http://www.ncbi.nlm.nih.gov/pubmed/19752606. 
14. $10.1111 / \mathrm{iju} .14011$

Hatano K, Tohyama N, Kodama T, Okabe N, Sakai M, Konoeda K. Current status of intensitymodulated radiation therapy for prostate cancer: History, clinical results and future directions. Int $\mathrm{J}$ Urol [Internet]. Blackwell Publishing; 2019 [cited 2020 May 22];26:775-84. Available from: https://onlinelibrary.wiley.com/doi/abs/10.1111/iju.14011.

15. 10.1016/j.radonc.2012.01.007

Hoskin PJ, Rojas AM, Bownes PJ, Lowe GJ, Ostler PJ, Bryant L. Randomised trial of external beam radiotherapy alone or combined with high-dose-rate brachytherapy boost for localised prostate cancer. Radiother Oncol [Internet]. Elsevier Ireland Ltd; 2012;103:217-22. Available from: http://dx.doi.org/10.1016/j.radonc.2012.01.007.

16. 10.1016/j.euf.2019.06.012

Ranasinghe WKB, Reichard CA, Bathala T, Chapin BF. Management of CT4 prostate cancer. Eur Urol Focus [Internet]. European Association of Urology; 2020;6:221-6. Available from: https://doi.org/10.1016/j.euf.2019.06.012.

17. Kasuya G, Ishikawa H, Tsuji H, Nomiya T, Makishima H, Kamada T, et al. Significant impact of biochemical recurrence on overall mortality in patients with high-risk prostate cancer after carbon-ion radiotherapy combined with androgen deprivation therapy. Cancer [Internet]. 2016 [cited 2018 May 20];122:3225-31. Available from:

https://www.ncbi.nlm.nih.gov/pmc/articles/PMC5094521/pdf/CNCR-122-3225.pdf.

18. Cahlon O, Hunt M, Zelefsky MJ. Intensity-modulated radiation therapy: supportive data for prostate cancer. Semin Radiat Oncol. 2008;18:48-57.

19. Yuan $P$, Wang S, Liu X, Wang X, Ye Z, Chen Z. The role of cystoprostatectomy in management of locally advanced prostate cancer: A systematic review. World J Surg Oncol. 2020;18:1-7.

\section{Figures}




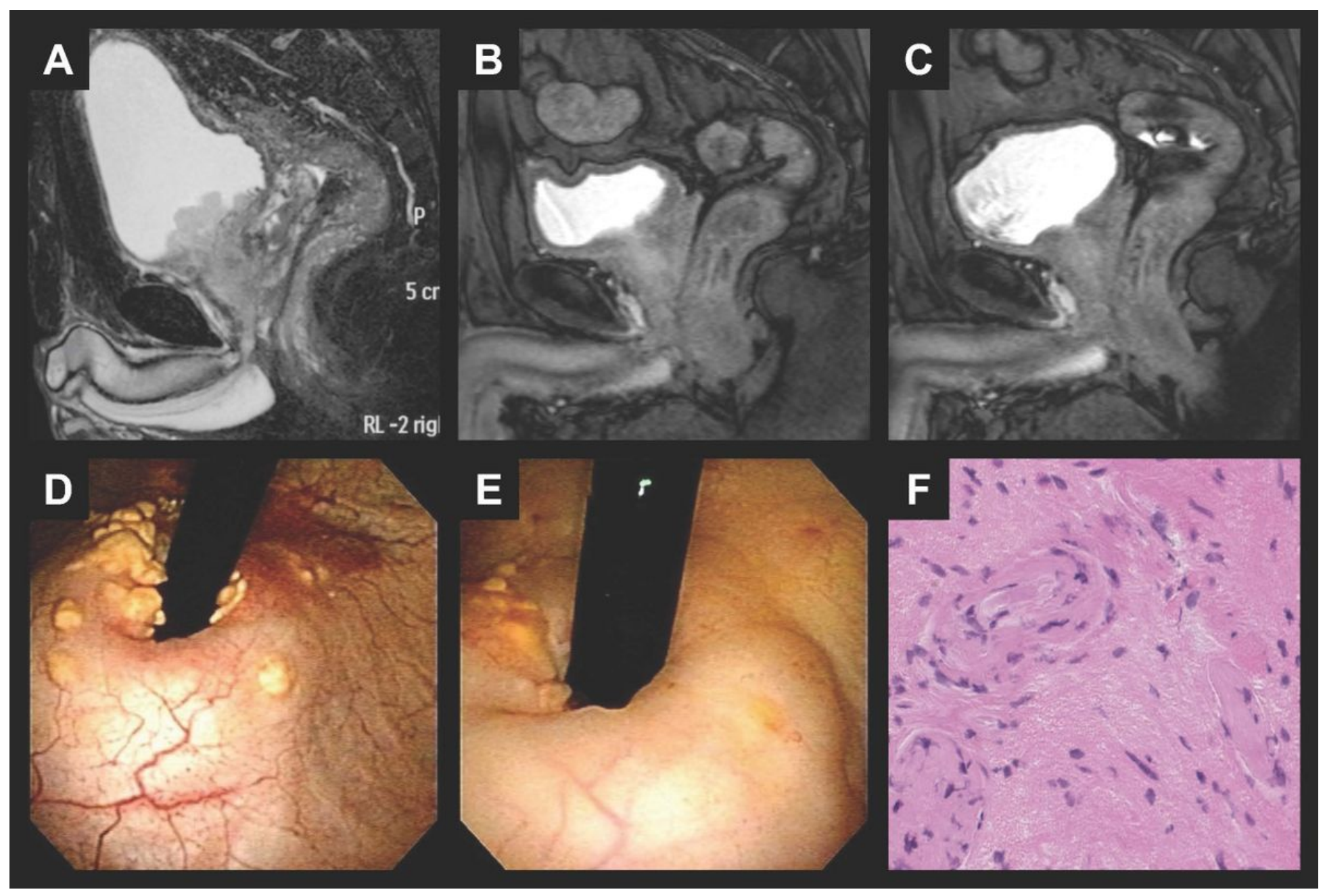

\section{Figure 1}

Magnetic resonance imaging (MRI), cystoscopic, and pathological findings of the representative case. Fat-saturated T2-weighted images (A) before ADT, (B) just before carbon ion radiotherapy (CIRT), and (C) 2 years after CIRT. Cystoscopic findings (D) just before CIRT and (E) one and half a year after CIRT. (F) Hematoxylin-Eosin stain of biopsy sample from the bladder lesion 2 years after CIRT in a high-power field. There were no malignant cells. 


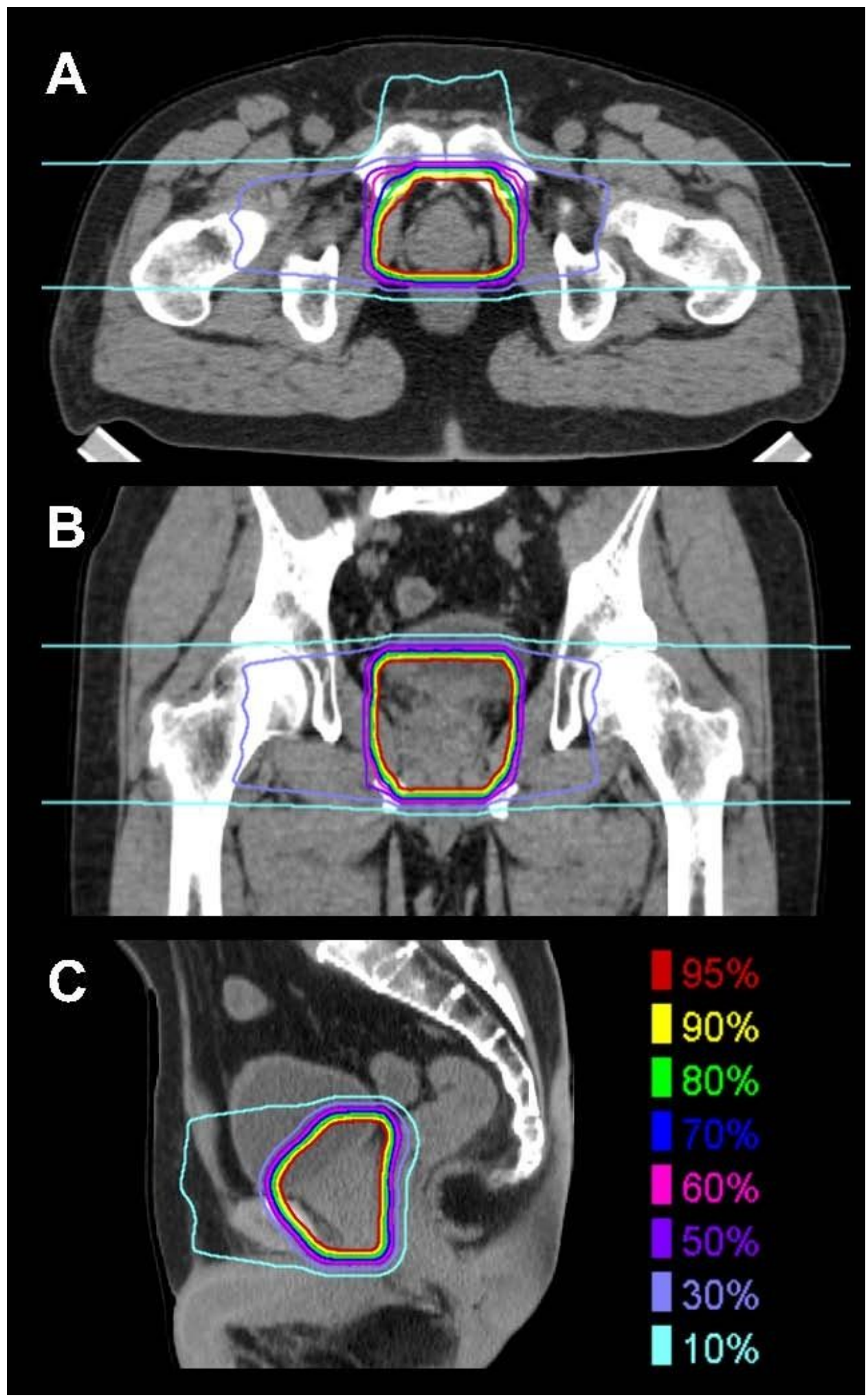

Figure 2

Dose distribution of carbon ion radiotherapy. (A) Axial, (B) coronal, and (C) sagittal images. 